\title{
Necrotizing Pneumonia: Diagnosis and Treatment Options
}

\author{
Brian D. Skidmore, BS ${ }^{1}$ and Veronica A. Arteaga, MD² \\ ${ }^{1}$ College of Medicine and ${ }^{2}$ Department of Medical Imaging \\ Banner-University Medical Center \\ University of Arizona \\ Tucson, AZ USA
}

\begin{abstract}
We present the case of a patient who was initially diagnosed with community-acquired pneumonia that was later discovered to have necrotizing changes. The case illustrates the challenges in diagnosing necrotizing pneumonia and the preferred treatment methods.
\end{abstract}

\section{Case Presentation}

History of Present Illness

The patient is a 51-year old woman who presents with right upper lobe pneumonia and a failed outpatient regimen of levofloxacin. She returned one week after being seen in the emergency department with worsening dyspnea, productive cough, and fever in addition to new symptoms of right chest pain and post-tussive emesis. The chest pain is stabbing in quality and constantly present. She denied any calf pain/swelling, previous history of deep venous thrombosis, or long trips or travels.

\section{Physical Exam}

Upon admission, blood pressure was $103 / 56$ with a pulse of 114 and respiratory rate of 18 . Her temperature was $38.1^{\circ} \mathrm{C}\left(100.5^{\circ} \mathrm{F}\right)$ but spiked at $39.5^{\circ} \mathrm{C}\left(103.1^{\circ} \mathrm{F}\right)$ and her $\mathrm{SpO}_{2}$ was $94.0 \%$ on room air. Her breathing was unlabored and her lungs were clear to auscultation bilaterally except for crackles in the right upper lung field. The remainder of the exam was unremarkable.

Laboratory and Imaging

A chest radiograph was initially obtained and showed a right upper lobe consolidation consistent with community-acquired pneumonia (Figure 1).

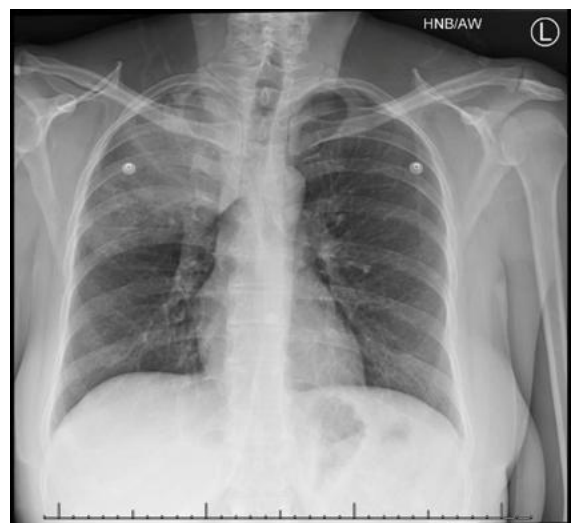

Figure 1. Chest radiograph showing right upper lobe consolidation with possible volume loss. 
One week later, a contrast-enhanced chest CT was performed and revealed a heterogeneously enhancing right upper lobe consolidation with cavitation and foci of air diagnostic of necrotizing pneumonia (Figure 2).

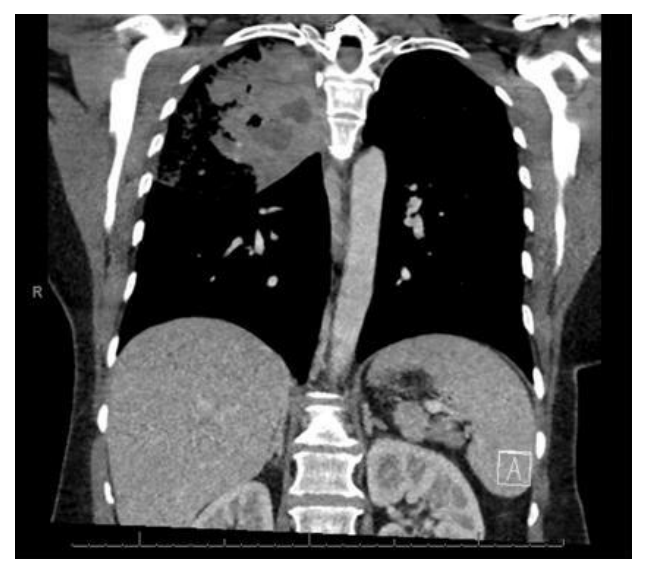

Figure 2. Contrast-enhanced chest CT showing right upper lobe pneumonic consolidation with peripheral enhancement, central necrosis, and small foci of air.

Laboratory studies revealed a markedly elevated C-reactive protein of $16.61 \mathrm{mg} / \mathrm{dL}$ and a white blood cell count of 18,000 cells/ $\mu \mathrm{L}$. In addition, the red blood cell count, hemoglobin, and hematocrit were all reduced with values of $3,390,000$ cells/ $\mu \mathrm{L}, 10.0 \mathrm{~g} / \mathrm{dL}$, and $31.0 \%$ respectively.

\section{Hospital Course}

A chest CT was ordered and the patient was diagnosed with necrotizing pneumonia. She was given IV vancomycin and piperacillin-tazobactam as empiric therapy. Tylenol was administered for fever management and steroids were deferred because her CURB-65 score for pneumonia severity was 0 .

Attention was then given to identifying the infectious agent. Blood and respiratory cultures were obtained and a TB test was ordered. The cultures showed no growth and the TB test was negative. A bronchoalveolar lavage showed a highly neutrophilic cell count, however no pathogen was ever identified.

Given improvement with empiric therapy, during her hospital course she was discharged on oral amoxicillin and clavulanate until follow up with pulmonary in outpatient 6 weeks later. Imaging at that time showed post inflammatory changes and no evidence of infection.

\section{Discussion}

Necrotizing pneumonia is a rare complication of bacterial lung infections affecting $4 \%$ of all patients with community-acquired pneumonia (1). The infection can be patchy, segmental, or involve the entire lung. While the pathogenesis of necrotizing pneumonia is not clearly defined, most studies indicate that it is either an inflammatory response to toxins produced by the pathogen or it is the result of associated vasculitis and venous thrombosis. Patients typically present with common symptoms of pneumonia such as fever, cough, shortness of breath, and chest pain but can also rapidly develop hemoptysis, septic shock, and respiratory failure as the necrosis progresses (2). Because necrotizing pneumonia is 
associated with increased morbidity and mortality, it is important to distinguish it from nonnecrotizing cases (3).

The diagnosis of necrotizing pneumonia may be difficult to make because of its similar presentation to non-necrotizing pneumonias and the limitations of standard chest radiographs. Chest radiographs may show an area of consolidation but are limited in identifying the extent of parenchymal disease (Figure 1) (2). Therefore, contrast-enhanced chest CT is an optimal exam for diagnosing necrotizing pneumonia. Disease may first appear as an in-homogeneously enhancing consolidation with focal areas of low attenuation (Figure 2). Foci of air may subsequently develop in these areas of hypo-enhancing necrotic tissue indicating cavitation (4).

Laboratory studies may also be helpful in diagnosing necrotizing pneumonia. When compared to pneumonias without a necrotizing component, patients with necrotizing pneumonia show more elevated white blood cell counts and inflammatory markers (1). In one study, patients with necrotizing pneumonia had an average WBC count of $14,970 / \mu \mathrm{L}$, an average ESR of $70 \mathrm{~mm} / \mathrm{h}$, and an average CRP of $18.8 \mathrm{mg} / \mathrm{dL}$. Average values for patients with non-necrotizing pneumonia were significantly lower at $10,130 / \mu \mathrm{L}, 48 \mathrm{~mm} / \mathrm{h}$, and 11.4 $\mathrm{mg} / \mathrm{dL}$ respectively $(\mathrm{p}<0.001)(3)$. These changes are also evident in the presented case with elevated WBC and CRP values of $18,000 / \mu \mathrm{L}$ and $16.61 \mathrm{mg} / \mathrm{dL}$.

Necrotizing pneumonia is initially treated with intravenously administered broad-spectrum antibiotics that should target pathogens that commonly cause necrotizing changes. The most common microbes are Staphylococcus aureus, Streptococcus pneumoniae, and Klebsiella pneumoniae, however several other bacteria species may also cause necrosis (Table 1) (2).

\title{
Table 1. Microbes that frequently cause necrosis
}

\author{
Gram positive bacteria \\ Staphylococcus aureus* \\ Staphylococcus epidermidis \\ Streptococcus pneumoniae* \\ Streptococcus pyogenes \\ Streptococcus viridans \\ Gram negative bacteria \\ A anetobacter baumannii \\ Escherichia coli \\ Haemophilus influenzae \\ Klebsiella pneumoniae* \\ Pseudomonas aeruginosa
}

*Denotes the pathogens that most commonly cause necrotizing pneumonia.

Transition to oral antibiotics may be considered for patients that show improvement (1). A more focused treatment plan should be initiated once a specific pathogen is identified, however this is only accomplished in approximately $26 \%$ of cases (3).

Surgical resection may also be considered for patients who show no progress on antibiotic therapy and continue to decline. However the optimal timing and indications for surgery are 
not clearly defined. The extent of the resection should always be as conservative as possible and commonly involves debridement or segmentectomy of the damaged tissue. In cases where the parenchyma is extensively affected, lobectomy or pneumonectomy may be required (2).

\section{References}

1. Nicolaou EV, Bartlett AH. Necrotizing pneumonia. Pediatr Ann. 2017;1;46(2):e65e68. [CrossRef] [PubMed]

2. Tsai YF, Ku YH. Necrotizing pneumonia: a rare complication of pneumonia requiring special consideration. Curr Opin Pulm Med. 2012;18(3):246-52. [CrossRef] [PubMed]

3. Seo $\mathrm{H}$, Cha SI, Shin $\mathrm{KM}$, et al. Clinical relevance of necrotizing change in patients with community-acquired pneumonia. Respirology. 2017;22(3):551-8. [CrossRef] [PubMed]

4. Walker CM, Abbott GF, Greene RE, Shepard JO, Vummidi D, Digumarthy SR. Imaging Pulmonary Infection: Classic Signs and Patterns. AJR Am J Roentgenol. 2014;202(3) 479-92. [CrossRef] [PubMed] 